\title{
Technological solution for the automatic replacement of the catalytic filaments in HWCVD
}

\author{
O. $\operatorname{Nos}^{1}$, P.A. Frigeri ${ }_{2}^{1}$ J. Bertomeu ${ }^{1}$. \\ ${ }^{1}$ Dept. de Física Aplicada i Òptica, Universitat de Barcelona \\ Barcelona-08028, Spain, Ph: +34934039221 , fax: +34934039219 \\ Email of corresponding author: oriol.nos@gmail.com
}

\begin{abstract}
The degradation of the catalytic filaments is the main factor limiting the industrial implementation of the hot wire chemical vapor deposition (HWCVD) technique. Up to now, no solution has been found to protect the catalytic filaments used in HWCVD without compromising their catalytic activity. Probably, the definitive solution relies on the automatic replacement of the catalytic filaments. In this work, the results of the validation tests of a new apparatus for the automatic replacement of the catalytic filaments are reported. The functionalities of the different parts have been validated using a $0.2 \mathrm{~mm}$ diameter tungsten filament under $\mu \mathrm{c}-\mathrm{Si}: \mathrm{H}$ deposition conditions.
\end{abstract}

\section{Keywords}

Hot wire chemical vapor deposition; catalytic chemical vapor deposition; silicon; filament lifetime; filament replacement. 


\section{Introduction}

Hot wire chemical vapor deposition (HWCVD) is a promising procedure for depositing and processing different thin film materials [1]. However, when the hot wires, also called catalytic filaments, are heated up to high temperature by Joule effect, they degrade [2]. The kind of degradation depends on the mixtures of gases used as deposition precursors. In the case of mixtures containing organic gases, the catalytic filaments are attacked by carburization [3], whereas for mixtures containing silicon precursors, like silane, the filaments are affected by silicides [2].

Several studies have been performed in order to understand the degradation processes affecting the catalytic filaments. In these studies, several solutions have been proposed to minimize the degradation of the filaments [4-11]. Although some progress has been achieved especially in increasing the structural life time [4-6, 8-10], i. e. the time for which the filament does not break, no definitive solution has been found so far, neither in stabilizing the filaments without affecting their catalytic activity [7], nor in a filament regenerating procedure which is not time consuming [11]. Furthermore, as we show in a parallel publication, the catalytic properties of the filaments are compromised since the catalytic surface starts to be affected by degradation [12]. The catalytic stability of the filament is guaranteed only during a short initial period of time when the decomposed radicals (for instance, Si radicals) form a solid solution with the catalytic material (for instance, a tungsten, W, filament) and no precipitation of alloys (like for instance $\mathrm{W}_{5} \mathrm{Si}_{3}$ or $\mathrm{WSi}_{2}$ ) has yet occurred. As we show in the same work, this catalytic life time can last no more than few hours, and, for filaments sufficiently thick it does 
not depend on the filament radius. This is much less than the structural life time, which can reach some hundred hours when using adequate protection devices. In this scenario, the automatic replacement of the catalytic filament seems to be the only way to overcome the main problem limiting the industrial implementation of the HWCVD technology, i.e. the short catalytic lifetime of the filament. This paper deals with a new HWCVD apparatus, rightly conceived for this purpose.

The manuscript is organized in the following way. Firstly, previous versions of such a HWCVD apparatus and their technological limitations are reviewed and analyzed. Secondly, the technological problems behind the filament replacement and their solutions are exposed. Finally, the validation tests of the new apparatus equipped with tungsten filaments subjected to a mixture of silane and hydrogen atmosphere are used to confirm the validity of the proposed solution.

\section{Historical background}

Already in 1994, Takaoki Sasaki from Fuji Electric Co, proposed a first apparatus for automatically changing the catalytic filament [13]. The idea is that a heat source body (corresponding to a catalytic filament) is supported between a sending part and a coiling part, sending out the heat source body from a sending part and winding it around to a coiling part. In the working examples given by Sasaki, the two reels forming the sending part and the coiling part are situated in the deposition chamber and are connected by means of two feedthroughs to motors located outside the chamber for driving the movement necessary to replace the degraded part of the filament. The main problems affecting the realization of his idea rely on the fact that the filament made of a 
refractory metal becomes very brittle at room temperature since silicon or carbon start dissolving in it. This makes it very difficult to wind the filament on the coiling part without breaking it. Furthermore, it is difficult to implement this idea to build a catalytic net composed of several filaments, because each filament needs two feedthroughs, which makes its realization economically prohibitive.

In 2006 Naoya Sotani, from Sanyo Electric Co disclosed his solutions to the above mentioned problems [14]. Sotani proposes the introduction of a heating unit to heat the used filament close to coiling part up to a temperature higher than its ductile-brittle transition, in order to avoid the risk of filament breakage. In [14] it is also proposed a way to create a catalytic net using a single catalytic filament covering a zigzag path around rollers placed along two distanced parallel lines in order to minimize the number of feedthroughs and motors necessary for the realization of the catalytic net.

As we will explain in the following section, it is possible to manage the replacement of the filament without having to wind it at a temperature higher than its ductile-brittle transition, which introduces an unnecessary complication in the realization of our system. In our opinion, the usage of a zigzag path to form a catalytic net is impracticable for several reasons. The usage of many rolls along a single catalytic filament introduces accumulative frictions which alter the catalytic filament tension. This can easily result in the local bending of the filament during the thermal expansion which follows its heating by Joule effect. In addition, the replacement velocity of the catalytic filament can become unsustainably high due to the fact that the catalytic filament portions forming the net are changed sequentially, one after the other. 
However, to our knowledge, all the ideas disclosed above are devoid of any test proving the feasibility of the proposed solution. The following section is devoted to explain the technological problems that we consider relevant for the realization of an apparatus for the automatic replacement of the catalytic filaments. Among them, we found the ones already reported by Sasaki and Sotani, namely the brittleness of the filament; as well as new problems arising from the presence of electrical and thermal contacts, and also other problems connected to the thermal expansion of the filament. The outline of the solutions implemented in the new apparatus is also given.

\section{Technological problems behind the filament replacement and their solutions}

\subsection{The brittleness of the filament}

As already mentioned, at room temperature the used portion of catalytic filament becomes extremely brittle since the first stage of dissolution of silicon or carbon in the catalytic material. This is mainly due to the precipitation-hardening of the refractory metal, which, in turn, is caused by the precipitation of the solute during the cooling of the wire. However, if the used portion of catalytic filament is wound around a reel with a sufficient large radius the internal stresses developed inside the catalytic filament are not sufficient to exceed the elastic limit of the material and no breaking occurs. However, for a typical filament radius of $0.5 \mathrm{~mm}$, the reel radius may result too big to be implemented. The trick is to use thinner filaments in such a way to reduce the reel radius. In fact, according to bending theory the internal stress magnitude suffered by the catalytic filament is directly proportional to the quotient between the radius of the filament and its radius of curvature, which in our case equals the radius of the reels. 
3.2 The electrical and thermal contact

A portion of filament becomes catalytically active by heating it up to high temperature by Joule effect. The active portion of catalytic filament is located between two brushes which act as electrical contacts and have to be kept perfectly clean to preserve the quality of the contact with the successive changes of the filament. This means that any kind of residual deposition must not reach the brushes. Otherwise, the electrical contact with the filament is degrading with the pass of the time. The electrical contact is also a thermal contact for the heated filament, what means that the portions of the filament next to the electrical contacts are colder than the central portion situated between the contacts. The kinetics of the degradation of the cold portions can be very different from the central portion. In case of the silicidation of tungsten filaments, the degradation of the cold portion is much more aggressive and rapid than the degradation of the central part, if the latter is heated at temperatures higher than $1700{ }^{\circ} \mathrm{C}$. The filament can degrade very quickly producing a premature breaking of the wire. However, also if any breaking does not occur, the enhanced degradation close to the electrical contact can act as nucleation centre for the silicidation. Fig. 1 shows the progress of the silicidation process in a system without any protection against nucleation of silidation which can arise close to the electrical contacts. The evolution of the silicidation is very different depending on the manner in which the filament is changed. In case the wire is replaced continuously, the silicide nucleates close to the sending reel and propagates rapidly in the direction of the coiling reel. In that case, after a short working time all the active part of the catalyst will be completely silicided. This is not the case if the filament is changed in a discontinuously way. 
To avoid any kind of deposition over the electrical contacts and also to avoid the premature degradation of the cold portions, we have developed a new kind of self cleaning cavity to surround and protect this fragile area of the system; the promising results after application are described in detail in sub-section 4.1. More details about this novel self-cleaning cavity device and its operating principle can be found in [15].

\subsection{Thermal expansion of the filament}

To avoid the catalytic filaments bending due to their lengthening it is necessary to implement a mechanism which tenses the catalytic filaments. This point is particularly important to guarantee good film homogeneity and the reproducibility of the HWCVD process. It becomes critical for all large area deposition systems implementing a continuous or discontinuous replacement of the catalytic filament.

A solution to this problem is based on the utilization of an electrical motor for controlling the torque over one of the two reels where the catalytic filament is wound, keeping tense the catalytic filament independently of its length. This could be a possible solution to the issue disclosed by Sasaki and Sotani. However, as we described in section 2, their inventions are not adequate to be scaled to large area catalytic nets. In the case of the apparatus disclosed by Sasaki, this is because of the excessive number of motors and feed-throughs needed, whereas, in the case of Sotani's, invention, the sequential modality to change the filament is not adequate.

The apparatus we conceived can be understood in a modular way. Each modulus manages a different filament which can form part of a net of various catalytic filaments. 
Each modulus includes a tension adjustment mechanism which keeps tense the corresponding filament in all operating situations. Since said modulus can work in vacuum conditions, only two motors are needed to change in parallel the whole net of catalytic filaments. Fig. 2 shows an outline of the new apparatus for the automatic replacement of the catalytic filament. More detail regarding this modular system can be found in [15]. The validation tests of the replacement mechanism are presented in subsection 4.3.

\section{Validation of the new apparatus}

4.1 Experimental tests of the self cleaning cavity protection device

The self-cleaning cavity protection device performance has been tested by its implementation next to one of the electrical contacts, while the other filament cold portion was protected by a simple cavity device [10]. The whole assembly permitted the filament displacement across both protection devices and the corresponding electric contacts. Hence, the tests have been performed using a single modulus of the developed automatic filament replacement mechanism. The experimental tests consisted in several series of deposition runs of about $20 \mathrm{~min}$ each, for an accumulated deposition time of more than $10 \mathrm{~h}$. After each deposition run, the used portion of the filament of $0.2 \mathrm{~mm}$ diameter, located between the two electric contacts, was wound on the coiling reel, thus being fully replaced by a new clean portion of the same filament unwound from the sending reel. Each deposition run took place introducing a silane and hydrogen gas

mixture in the vacuum chamber at a pressure of about $10 \mathrm{~Pa}$ with a silane dilution of 
about $10 \%$ in volume with respect to the introduced gas mixture. The temperature of the filament during the deposition run was about $1850^{\circ} \mathrm{C}$.

Fig. 3 shows the aspect of the inner walls of either the simple cavity or the self cleaning cavity, photographed after a certain amount of accumulated deposition time: less than $3 \mathrm{~h}$ for the simple cavity, and more than $10 \mathrm{~h}$ for the self cleaning cavity. The simple cavity (Fig. 3.a) needed to be cleaned very often as it was rapidly filled with silicon material that peeled off from its inner walls. On the other hand, the inner walls of the self cleaning cavity (Fig. 3.b) which envelops the filament zone located next to the other electrical contact were perfectly clean, meaning that any kind of deposition reaching the corresponding electrical contact was prevented.

The effects of silicidation on the different regions of the portion of filament used in a single run have been studied by means of SEM images, shown in Fig. 4, and EDX analysis. Fig. 4.a shows the cold portion protected by the simple cavity device. This portion presented a large density of cracks and an outer corona which has been identified by the EDX analysis as a rich silicon $\mathrm{WSi}_{2}$ silicide phase. Fig. 4.b shows the central region of the filament where the outer corona has been identified with the initial formation of $\mathrm{W}_{5} \mathrm{Si}_{3}$ silicide. Finally, Fig. 4.c corresponds to the cold portion that has been protected with the self cleaning cavity. It can be observed that the filament is in almost perfect condition, and no silicon has been detected with the EDX analysis in this region of the filament. The cold portion protected by the self cleaning cavity was found in a better condition than the central part of the filament.

4.2 Experimental tests of the automatic filament replacement mechanism 
We monitored the power per unit length dissipated by the catalytic filament during several deposition runs, in order to verify that the replacement of a portion of used catalytic filament leads the new HWCVD apparatus back to the same initial conditions observed before the deposition took place. Fig. 5 shows the result for a sequence of six deposition runs. When the power per unit length $\mathrm{P}_{\mathrm{f}}$ was about $2.6 \mathrm{~W} / \mathrm{cm}$, the approximate temperature of the catalytic filaments was $1850{ }^{\circ} \mathrm{C}$. During these time periods, the precursor gas mixture entered into the vacuum chamber and the thin film deposition took place. The period times when $\mathrm{P}_{\mathrm{f}}$ was about $0.2 \mathrm{~W} / \mathrm{cm}$ corresponded to the change of the portion of catalytic filament located between both electrical contacts. When the deposition took place, $\mathrm{P}_{\mathrm{f}}$ slowly decreased, maybe due to the degradation of the catalytic filament, in particular on the cold portion covered by the simple cavity. However, the change of the used portion of catalytic filament took back $\mathrm{P}_{\mathrm{f}}$ to the same initial value observed at the start of each deposition run. The electric power fluctuations were less than $\pm 1 \%$ for each deposition period time.

\section{Conclusion}

This research has addressed the traditional problems associated with the automatic replacement of the catalytic filament used in the HWCVD technique, and showcases our own technology to overcome the stumbling blocks for automatic filament replacement assembly. We have shown that the main problems arise from three distinct aspects which make difficult the filament replacement: the enhanced brittleness of the used filament, the criticality of the electrical contacts region, and the thermal expansion of the filament. We showed these aspects can be solved by using: thin 
catalytic filaments to facilitate the winding of the used filament, a self cleaning cavity to protect and maintain clean the region close to the electrical contact, and a modular tension adjustment mechanism, to keep tense each filament independently. Two different tests have been carried out to validate the functioning of the new apparatus. The first one showed that the area protected by the self-cleaning cavity were uncontaminated by silicon deposition after more than $10 \mathrm{~h}$ of accumulated deposition time under $\mu \mathrm{c}-\mathrm{Si}: \mathrm{H}$ deposition conditions. The cold portion of filament located inside the self cleaning cavity was also protected and was found in better conditions than the hotter central part of the filament. The second test used the monitoring of the power per unit length dissipated by the filament to show the new apparatus recovered its initial conditions after each cycle of deposition followed by the filament change. These results proved the effectiveness of the new HWCVD apparatus to guarantee the reproducibility and stability of the catalytic filament conditions in a HWCVD process.

\section{Acknowledgements}

This research has been supported by the Spanish Ministerio de Ciencia e Innovación and the European Regional Development Fund through the projects AMIC (ENE2010-21384-C4-03) and INNDISOL (IPT-420000-2010-006).

\section{References}

1. $\quad$ R.E.I Schropp, Thin Solid Films 517 (2009) 3415.

2. J. Doyle, R. Roberson, G.H. Lin, M.Z. He, A. Gallager, J. Appl. Phys. 64 (1988) 3215. 
3. S. Okoli, R Haubner, B. Lux, Surface and Coatings technology 47 (1991) 585.

4. $\quad$ K. Ishibashi, Thin Solid Film 395 (2001) 55.

5. K. Ishibashi, M. Karasawa, G. Xu, N. Yokokawa, I. Manabu, A. Masuda, H. Matsumura, Thin Solid Films 430 (2003) 58.

6. D. Grunsky, M, Kupich, B. Hofferberth, B. Schroeder, Thin Solid Films 501 (2006) 322.

7. K. Honda, O. Keisuke, H. Matsumura, Thin Solid Films 516 (2008) 826.

8. D. Knoesen, C. Arendse, S. Halindintwali, T. Muller, Thin Solid Films 516 (2008) 822.

9. N. Kniffler, A. Pflueger, D. Scheller, B. Schroeder, Thin Solid Films 517 (2009) 3424.

10. P.A. Frigeri, O. Nos, S. Bengoechea, C. Frevert, J.M. Asensi, J. Bertomeu, Thin Solid Films 517 (2009) 3427.

11. C.H.M. van der Werf, H. Li, V. Verlaan, C.J. Oliphant, R. Bakker, Z.S. Houweling, R.E.I Schropp, Thin Solid Films 517 (2009) 3431.

12. P.A. Frigeri, O. Nos, J. Bertomeu, These proceedings.

13. Fuji Electric Co Ltd, Thin Film Forming Apparatus, T. Sasaki, JP7254566 (A), October 3, 1995.

14. Sanyo Electric Co Ltd, cat-CVD Device and Filament Replacement Method, N. Sotani, JP2008140945 (A), June 19, 2008.

15. Universitat de Barcelona, Device and method for hot-wire chemical vapour deposition, P.A. Frigeri, O. Nos, J. Bertomeu, WO/2013/093165, June 27, 2013. 


\section{Figure Captions}

Fig. 1: Effect of the filament change modality (continuous or discontinuous) over the propagation of the silicide parts nucleating close to the cool portion of the filament. The silicide parts are represented by grey arrows.

Fig. 2: Outline of the new apparatus for the automatic replacement of the catalytic filament. The apparatus is conceived in a modular way. The following parts are indicated: a. the filament; b. protection device for the protection of the filament cold portion (ex: self cleaning cavity protection device); c. electrical contact; d. tension adjustment mechanism, e. reception reel also called in the text coiling part; f. motor.

Fig. 3: Photographs of the internal walls of the simple cavity, which was conceived in two shells (top), and of the self cleaning cavity, which was photographed by a lateral aperture normally not accessible (bottom). The simple and the self cleaning cavities were photographed after an accumulated deposition time of $3 \mathrm{~h}$ and $10 \mathrm{~h}$ respectively.

Fig. 4: SEM images of cross sections of differently located portions of a used filament taken after a deposition run of 20 min: a. cold portion protected by the simple cavity; $b$. unprotected central hot portion; c. cold portion protected by the self cleaning cavity.

Fig. 5: Electrical power per unit length, $\mathrm{P}_{\mathrm{f}}$, dissipated by the filament as a function of time, monitored during 6 consecutive deposition cycles. Once every two deposition cycles, the electric power, which was supplying the filament, was switched off. 
Fig.1
Continuous
Discontinuous

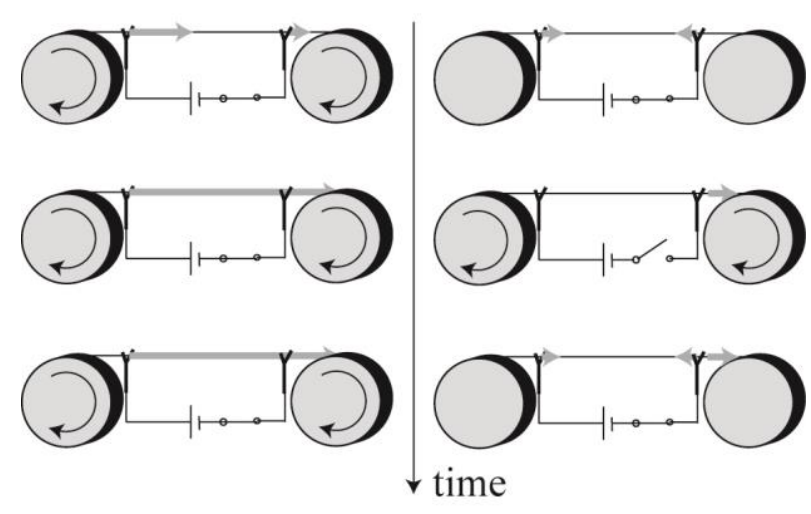

Fig. 2

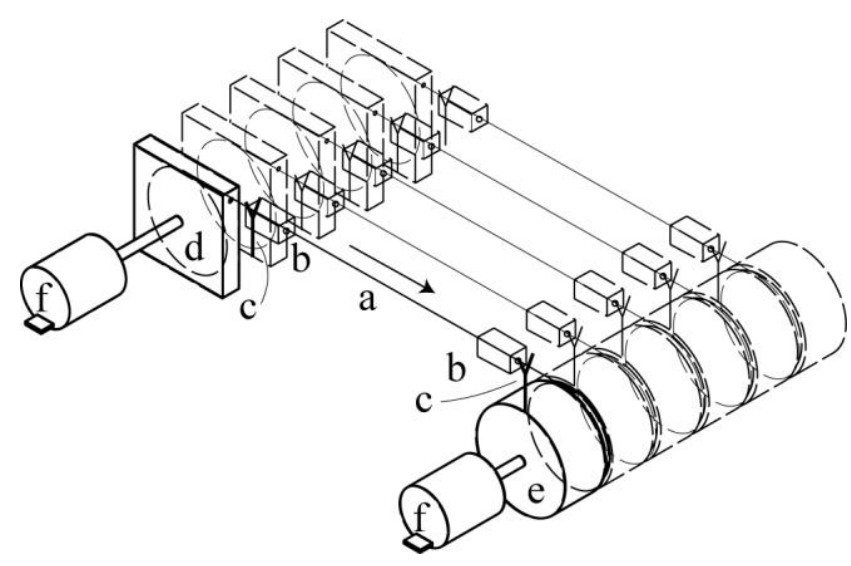


Fig. 3
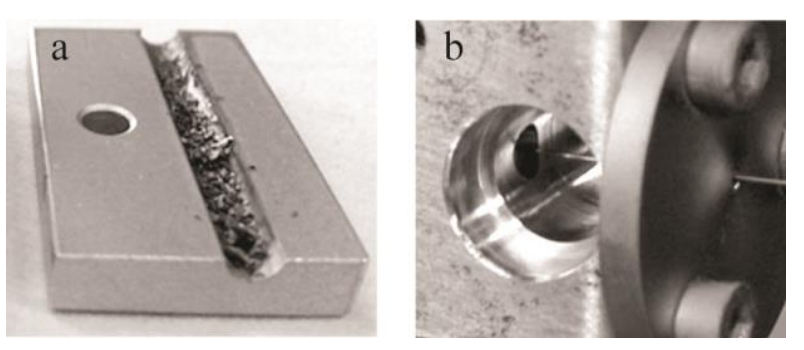

Fig. 4

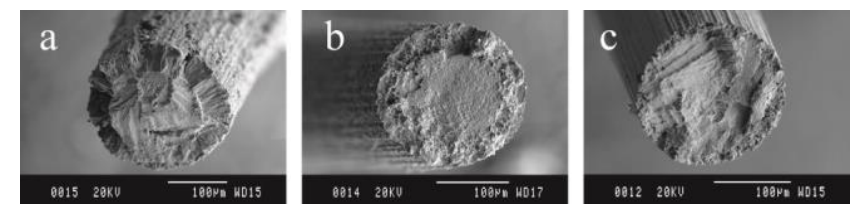

Fig. 5

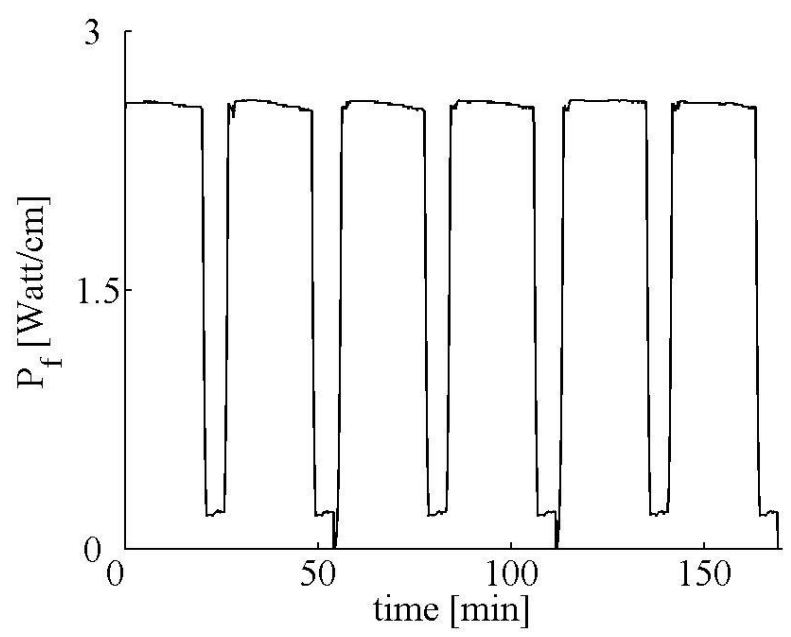

